\title{
Nonlinear Refractive Index Measurement Utilizing Bistable Behavior of Double Coupling Optical Fiber Ring Resonator
}

\author{
Lei YANG*, Wei PAN, Bin LUO, and Lianshan YAN \\ Center for Information Photonics \& Communications, School of Information Science and Technology, Southwest \\ Jiaotong University, Chengdu, 610031, China \\ ${ }^{*}$ Corresponding author: Lei YANGＥ-mail: yanglei19811212@gmail.com
}

\begin{abstract}
A novel approach for measuring the nonlinear refractive index of an optical fiber utlizing the bistable behavior of the double coupling optical fiber ring resonator was proposed and investigated. The switch-off or switch-on power decreases with an increase in the nonlinear refractive index $n_{2}\left(\mathrm{~m}^{2} / \mathrm{W}\right)$, and the dependence of swith-off or switch-on power on the nonlinear refractive index was analyzed numerically. Simulation results showed that the switch-off power and switch-on power (in dBW) decreased linearly with $\log _{10}\left(n_{2}\right)$ in a 100 -m-length fiber ring resonator, when $n_{2}$ changed from $3.2 \times 10^{-20} \mathrm{~m}^{2} / \mathrm{W}$ to $2.5 \times 10^{-17} \mathrm{~m}^{2} / \mathrm{W}$ or nearly $n_{2}=3.2 \times 10^{-20} \mathrm{~m}^{2} / \mathrm{W}$. These mean that high accuracy as well as large-scale nonlinear refractive index measurement can be achieved by the proposed approach.
\end{abstract}

Keywords: Bistable behavior, nonlinear refractive index, optical fiber ring resonator

Citation: Lei YANG, Wei PAN, Bin LUO, and Lianshan YAN, "Nonlinear Refractive Index Measurement Utilizing Bistable Behavior of Double Coupling Optical Fiber Ring Resonator," Photonic Sensors, 2015, 5(1): 79-83.

\section{Introduction}

The optical nonlinear resonator has been attracting greatly attention due to its variety of possible operating regimes, including bistability, period-doubling, or chaos [1]. Bistability commonly refers to a state in which the output intensity of a device may take two different values for the same input intensity depending on the initial conditions [2]. Optical bistabiltiy has been studied widely over the past years, and numberous applications such as differential amplification, optical thresholding, or all-optical switching have been demonstrated [3-6]. This interesting behavior originates in an optical Kerr effect that the refractive index of an optical fiber is changed depending on the optical intensity.
Therefore, nonlinear refractive index information of the fiber can be extracted from the bistablity characteristics in the optical nonlinear resonator.

In this work, we proposed and investigated a nonlinear refractive index measurement approach utilizing the bistable behavior of the double coupling optical fiber ring resonator structure. Compared to one of major conventional methods which uses the continuous wave $(\mathrm{CW})$ probe light that composes a Mach-Zehnder interferometer for obtaining the phase shift and optical pump pulses that are produced by cross phase-modulation in the probe light $[7,8]$, our approach can avoid ultra-high pump power and mitigate the large error in measuring the interference fringe shift. Addtionally, the simulation results showed that high accuracy as well as large-

Received: 30 October 2014 / Revised version: 12 December 2014

(C) The Author(s) 2014. This article is published with open access at Springerlink.com

DOI: $10.1007 / \mathrm{s} 13320-014-0228-6$

Article type: Regular 
scale nonlinear refractive index measurement could be achieved by this proposed approach.

\section{Modeling}

A schematic diagram of the double coupling optical fiber ring resonator is depicted in Fig. 1. The input light $E_{i}$ is launched into the double coupling optical fiber ring resonator through an optical coupler (OC1). The light from Port 4 of OC1 $\left(E_{t 1}\right)$ propagates a half-round trip in the ring and then is coupled by the optical coupler (OC2). After OC2, part of the light $\left(E_{d}\right)$ is guides out of the resonator from Port 7 of OC2, and part of the input light $\left(E_{t 2}\right)$ after propagating in the resonator is coupled with the circulated light in the $\mathrm{OC} 1$.

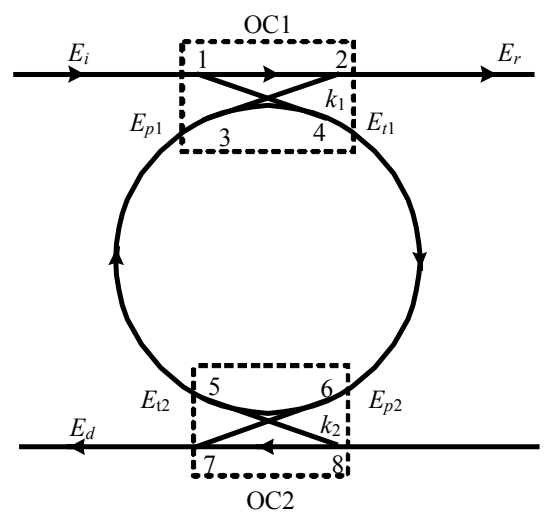

Fig. 1 Schematic diagram of the double coupling optical fiber ring resonator.

In the double coupling optical fiber ring resonator system, the output optical fields from OC1 and $\mathrm{OC} 2$ can be expressed as

$$
\begin{aligned}
& {\left[\begin{array}{l}
E_{t 1}(t) \\
E_{r}(t)
\end{array}\right]=M_{1}\left[\begin{array}{l}
E_{\text {in }}(t) \\
E_{p 1}(t)
\end{array}\right]} \\
& {\left[\begin{array}{l}
E_{t 2}(t) \\
E_{d}(t)
\end{array}\right]=M_{2}\left[\begin{array}{c}
0 \\
E_{p 2}(t)
\end{array}\right]}
\end{aligned}
$$

where $M_{1}$ and $M_{2}$ are the transmission matrices of $\mathrm{OC} 1$ and $\mathrm{OC} 2$, respectively, which are given by

$$
\begin{gathered}
M_{1}=\sqrt{1-p_{1}}\left[\begin{array}{ll}
\mathrm{j} \sqrt{\kappa_{1}} & \sqrt{1-\kappa_{1}} \\
\sqrt{1-\kappa_{1}} & \mathrm{j} \sqrt{\kappa_{1}}
\end{array}\right] \\
M_{2}=\sqrt{1-p_{2}}\left[\begin{array}{cc}
\mathrm{j} \sqrt{\kappa_{2}} & \sqrt{1-\kappa_{2}} \\
\sqrt{1-\kappa_{2}} & \mathrm{j} \sqrt{\kappa_{2}}
\end{array}\right]
\end{gathered}
$$

where $p_{n}(n=1,2)$ and $k_{i}(i=1,2)$ are the fractional intensity loss and intensity coupling coefficient of the coupler, respectively.

Considering the propagating effect in the ring, $E_{p 1}(t)$ and $E_{p 2}(t)$ are given by

$$
\begin{gathered}
E_{p 1}(t)=E_{t 2}\left(t-\tau_{r}\right) \tau \operatorname{ex}\left[-\mathrm{j} \phi_{2}\left(t-\tau_{r}\right)\right] \\
E_{p 2}(t)=E_{t 1}\left(t-\tau_{r}\right) \tau \operatorname{ex}\left[-\mathrm{j} \phi\left(t-\tau_{r}\right)\right] \\
\phi_{1}(t)=\phi_{L 1}+\phi_{\mathrm{NL} 1}(t)=\phi_{L 1}+\pi n_{2} L\left|E_{t 1}(t)\right|^{2} / \lambda \\
\phi_{2}(t)=\phi_{L 2}+\phi_{\mathrm{NL} 2}(t)=\phi_{L 2}+\pi n_{2} L\left|E_{t 2}(t)\right|^{2} / \lambda
\end{gathered}
$$

where $\tau_{r}=n_{0} L / c$ is the half round-trip time of the ring, $L$ is the length of the ring, $n_{2}$ is the nonlinear refractive index of the fiber in the ring, and $\lambda$ is the wavelength of the input light.

Therefore, the two output optical fields at $\mathrm{OC} 1$ $E_{r}(t)$ and $\mathrm{OC} 2 E_{d}(t)$ can be finally expressed as follows:

$$
\begin{gathered}
E_{r}(t)=\sqrt{1-p_{1}}\left\{\sqrt{1-k_{1}} E_{\text {in }}(t)+\mathrm{j} \sqrt{k_{1}} E_{p 1}(t)\right\} \\
E_{d}(t)=\mathrm{j} \sqrt{1-p_{2}} \sqrt{k_{2}} E_{p 2}(t) .
\end{gathered}
$$

\section{Numerical analysis and discussion}

Here, we focus on the numerical investigation into the bistable behavior of the double coupling optical fiber ring resonator. So, we assume that the input light is a Guassian pulse with the peak power of $35 \mathrm{~W}$ and full width at the half maximum intensity (FWHM) of $1.44 \mathrm{~ms}$. Firstly, we consider a common case and assume the optical fiber with linear and nonlinear refractive indices $n_{0}=1.45$ and $n_{2}=3.2 \times 10^{-20} \mathrm{~m}^{2} / \mathrm{W}$, respectively, and an effective mode area $S_{\text {effs }}$ is $30 \mu \mathrm{m}^{2}$. It is also assumed that the length $L$ of the ring fiber is $100 \mathrm{~m}$, the loss $a$ is $0.2 \mathrm{~dB} / \mathrm{km}$, the operation wavelength $\lambda=1550 \mathrm{~nm}$, $k_{1}=0.9, k_{2}=0.75$, and $p_{1}=p_{2}=0.1$.

Figures 2(a) and 2(b) show the bifurcation diagrams at two outputs of the ring (Port 2 and Port 7 shown in Fig. 1), respectively. As shown in Fig.2, the bistable behavior is observed at both output ports, which means that we can use the bistable behavior of any output port to carry out nonlinear refractive index measurement. 

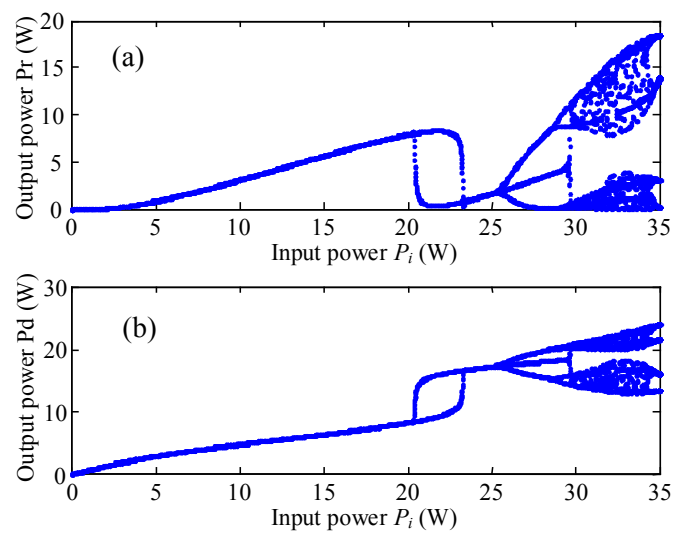

Fig. 2 Bifurcation diagrams of the double coupling optical fiber ring resonator at output $P_{r}$ and $P_{d}$, respectively.

In fact, the bistable behavior of the double coupling optical fiber ring resonator is sensitive to the system's parameters, such as the nonlinear refractive index of the fiber. Therefore, we can utilize this effect to measure the nonlinear refractive index of the fiber by building up a ring resonator. Figure 3 shows the bistable behavior variation of the fiber ring resonator when different type fibers are applied. The corresponding nonlinear refractive indices in Figs. 3(a), 3(b), 3(c), and 3(d) are $n_{2}=3.2 \times 10^{-20} \mathrm{~m}^{2} / \mathrm{W}, \quad 2 \times 10^{-19} \mathrm{~m}^{2} / \mathrm{W}, \quad 4.2 \times 10^{-18}$ $\mathrm{m}^{2} / \mathrm{W}$, and $2.5 \times 10^{-17} \mathrm{~m}^{2} / \mathrm{W}$, respectively. As shown in Fig. 3, under the bistable processing, the switch-off power and switch-on power decrease with an increase in the nonlinear refractive index. Therefore, the nonlinear refractive index information of the fiber in the ring can be extracted from the characteristics in the resonator. To obtain more details about the relationship between the switch-off or switch-on power and nonlinear refractive index, we applied more kinds of fibers in the ring resonator, and the simulation results are shown in Fig. 4. According to Fig. 4, the switch-off power and switch-on power (in dBW) decreased linearly with $\log _{10}\left(n_{2}\right)$. Taking the switch-on power as an example (shown in Fig. 5), the switch-on power (in $\mathrm{dBW}$ ) and the logarithm of nonlinear refractive index $n_{2}^{\prime}=\log _{10}\left(n_{2}\right)$ can be explicitly expressed as

$$
P_{\text {in }}(\mathrm{dBW})=-0.9711 \times n_{2}^{\prime}-40.8875 .
$$
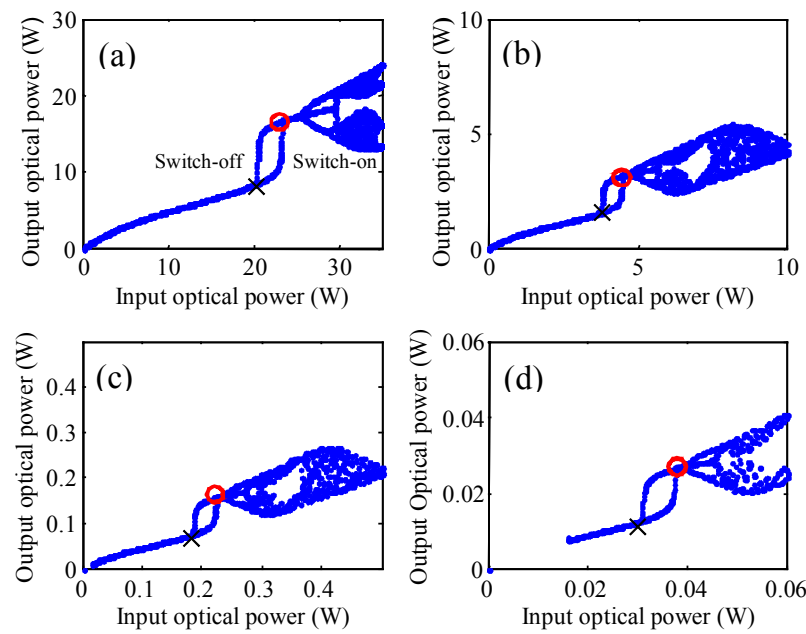

Fig. 3 Bifurcation diagrams when the fibers with different nonlinear refractive indices are applied: (a) $n_{2}=3.2 \times 10^{-20} \mathrm{~m}^{2} / \mathrm{W}$, (b) $n_{2}=2 \times 10^{-19} \mathrm{~m}^{2} / \mathrm{W}, \quad$ (c) $n_{2}=4.2 \times 10^{-18} \mathrm{~m}^{2} / \mathrm{W}, \quad$ and (d) $n_{2}=2.5 \times 10^{-17} \mathrm{~m}^{2} / \mathrm{W}$.

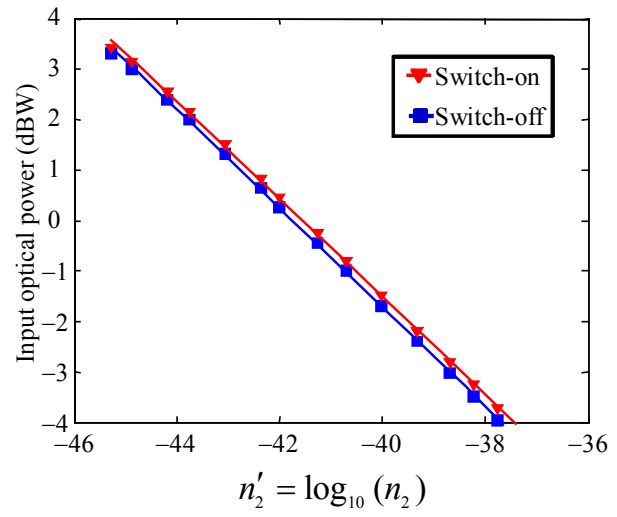

Fig. 4 Relationship between the switch-off and switch-on power and the nonlinear refractive index.

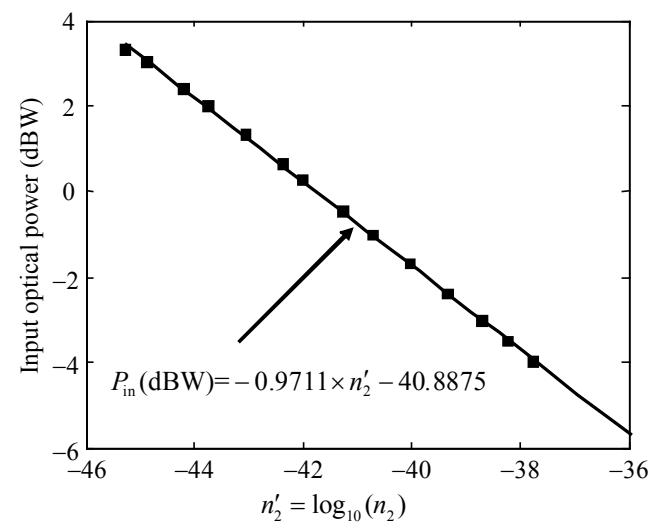

Fig. 5 Relationship between the switch-on and $n_{2}^{\prime}$ when $n_{2}$ changes from $3.2 \times 10^{-20} \mathrm{~m}^{2} / \mathrm{W}$ to $2.5 \times 10^{-17} \mathrm{~m}^{2} / \mathrm{W}$.

According to (11), we can calculate the nonlinear refractive index $n_{2}$ by simply detecting the 
switch-off or switch-on power value. As shown in Fig. 5, the relationship between the switch-on power and $n_{2}$ satisfies (11) in the large area (from $3.2 \times 10^{-20} \mathrm{~m}^{2} / \mathrm{W}$ to $2.5 \times 10^{-17} \mathrm{~m}^{2} / \mathrm{W}$ ), which means large-scale nonlinear refractive index measurement can be achieved by this approach.

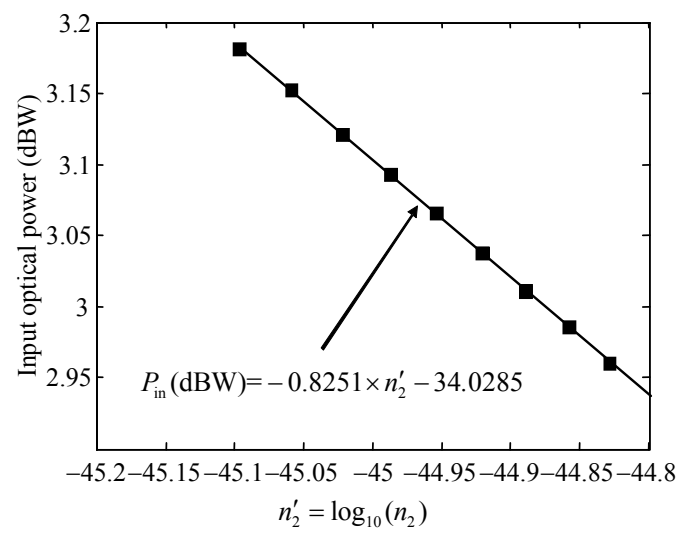

Fig. 6 Relationship between the switch-on and $n_{2}^{\prime}$ when $n_{2}$ changes from $2.6 \times 10^{-20} \mathrm{~m}^{2} / \mathrm{W}$ to $3.4 \times 10^{-20} \mathrm{~m}^{2} / \mathrm{W}$.

In some applications, the nonlinear refractive index of the optical fiber should be measured precisely, such as the chaos system, sensing, and all optical signal processing [9-11]. From the point of view of the application, we further investigated the relationship between the switch-on power and the nonlinear refractive index when the nonlinear refractive index varied in a small range. Figure 6 shows the relationship between the switch-on power and the logarithm of the nonlinear refractive index $n_{2}^{\prime}=\log _{10}\left(n_{2}\right)$ when $n_{2}$ changed from $2.6 \times 10^{-20} \mathrm{~m}^{2} / \mathrm{W}$ to $3.4 \times 10^{-20} \mathrm{~m}^{2} / \mathrm{W}$. As shown in Fig. 6, the swtich-on power and $n_{2}^{\prime}$ satisfied the linear relationship as well, when $n_{2}$ changed in a small range. So, the high accuracy nonlinear refractive index measurement can be obtained by this approach as well.

\section{Conclusions}

We proposed and numerically analyzed a nonlinear refractive index measurement approach utilizing the bistable behavior of the double coupling optical fiber ring resonator. The simulation results showed that the switch-off power and switch-on power (in $\mathrm{dBW}$ ) decreased linearly with $\log _{10}\left(n_{2}\right)$ in a 100-m-length fiber ring resonator, when $n_{2}$ changed from $3.2 \times 10^{-20} \mathrm{~m}^{2} / \mathrm{W}$ to $3.5 \times 10^{-15} \mathrm{~m}^{2} / \mathrm{W}$ or nearly $n_{2}=3.2 \times 10^{-20} \mathrm{~m}^{2} / \mathrm{W}$. Therefore, high accuracy as well as large-scale nonlinear refractive index measurement can be achieved by this proposed approach.

Open Access This article is distributed under the terms of the Creative Commons Attribution License which permits any use, distribution, and reproduction in any medium, provided the original author(s) and source are credited.

\section{References}

[1] V. Van, T. A. Ibrahim, P. P. Absil, F. G. Johnson, R. Grover, and P. Ho, "Optical signal processing using nonlinear semiconductor microring resonators," IEEE Journal of Selected Topics in Quantum Electronics, 2002, 8(3): 705-713.

[2] A. L. Steele, "Optical bistability, instabilities and power limiting behaviour from a dual nonlinear optical fibre loop mirror resonator," Optics Communications, 2004, 236(1-3): 209-218.

[3] M. Mimuro, S. Yamauchi, K. Suzuki, and Y. Imai, "Proposal for nonlinear refractive index measurement using spectral ratio in modulated OFRR dynamics," Optics Communications, 2008, 281(3): 469-473.

[4] Y. C. Lin, M. Mao, Y. Lin, H. Lin, C. Lin, and L. A. Wang, "All-optical switching in GaAs microdisk resonators by a femtosecond pump-probe technique through tapered-fiber coupling," Optics Letters, 2014, 39(17): 4998-5001.

[5] G. Genty, M. Lehtonen, and H. Ludvigsen, "Optical bistability and signal processing in a microstructured fiber ring resonator," Applied Physics B, 2005, 81(2-3): 357-362.

[6] K. Saleh, O. Llopis, and G. Cibiel, “Optical scattering induced noise in fiber ring resonators and optoelectronic oscillators," Journal of Lightwave Technology, 2013, 31(9): 1433-1446.

[7] L. Zhang, P. Lu, L. Chen, C. Huang, D. Liu, and S. Jiang, "Optical fiber strain sensor using fiber resonator based on frequency comb Vernier spectroscopy," Optics Letters, 2012, 37(13): 2622-2624.

[8] G. Yin, S. Lou, and H. Zou, "Refractive index sensor with asymmetrical fiber Mach-Zehnder interferometer 
based on concatenating single-mode abrupt taper and core-offset section," Optics and Laser Technology, 2013, 45: 294-300.

[9] C. Jauregui, J. M. L. Higuera, A. Cobo, O. M. Conde, and J. Zubía, "Multiparameter sensor based on a chaotic fiber-ring resonator," Journal of the Optical Society of America B, 2006, 23(10): 2024-2031.

[10] T. Suzuki, S. Wei, and Y. Imai, "Dependence of output power in modulated optical fiber ring resonator on nonlinear refractive index," Optical Review, 2010, 17(3): 327-330.

[11] Y. Imai, H. Yokota, and S. Yamauchi, "Measurements of nonlinear refractive index of optical fiber by using multiple interference in OFRR," Photonics and Optoelectronics, 2013, 2(3): 78-83. 